\title{
Research Article \\ Existence for Competitive Equilibrium by Means of Generalized Quasivariational Inequalities
}

\author{
I. Benedetti, ${ }^{1}$ M. B. Donato, ${ }^{2}$ and M. Milasi ${ }^{2}$ \\ ${ }^{1}$ Department of Mathematics and Computer Science, University of Perugia, 06123 Perugia, Italy \\ ${ }^{2}$ Department of Mathematics and Computer Science, University of Messina, 98166 Messina, Italy
}

Correspondence should be addressed to M. Milasi; mmilasi@unime.it

Received 3 March 2012; Revised 8 December 2012; Accepted 18 December 2012

Academic Editor: Sining Zheng

Copyright (C) 2013 I. Benedetti et al. This is an open access article distributed under the Creative Commons Attribution License, which permits unrestricted use, distribution, and reproduction in any medium, provided the original work is properly cited.

\begin{abstract}
A competitive economic equilibrium model integrated with exchange, consumption, and production is considered. Our goal is to give an existence result when the utility functions are concave, proper, and upper semicontinuous. To this aim we are able to characterize the equilibrium by means of a suitable generalized quasi-variational inequality; then we give the existence of equilibrium by using the variational approach.
\end{abstract}

\section{Introduction}

In this paper a competitive economic equilibrium model integrated with exchange, consumption, and production is considered. In 1874, Walras [1] laid the foundations for the study of the general equilibrium theory. However, the first rigorous result on the existence of general equilibrium is due to Wald; see, for example, [2]. After this pioneering work, several authors (including [3-5]), stimulated by the advances in linear programming, activity analysis, and game theory, obtained equilibrium existence results. In particular, Arrow and Debreu considered the application of fixed point theory to equilibrium problems, establishing the existence of an equilibrium in an abstract economy that included production and consumption.

Recently, an alternative approach to the study of general economic equilibrium has been considered in terms of variational inequalities. Many economic equilibrium models, including the general equilibrium model of Arrow-Debreu, can be formulated as variational inequalities and/or complementarity problems. Variational theory was introduced in the early 1960s with the works of Fichera and Stampacchia; they study equilibrium problems arising from elastoplastic theory and from mechanics. Subsequently this theory was applied in different kinds of equilibrium problems and, now, represents a powerful tool for the study of a large class of equilibrium problems arising in mechanics, physics, optimization and control theory, operations research, and several branches of engineering sciences. For the state of art about this topic, we refer the reader to [6-14] and the bibliography therein.

In particular, Jofré et al. in [15] studied the competitive equilibrium by means of a variational inequality, which involves the Lagrange multipliers. Our purpose in this paper is to study the economic competitive equilibrium through a different variational method. In fact, we can characterize the equilibrium by means of a generalized quasi-variational inequality without using the method of Lagrange multipliers. A competitive equilibrium as solution of a suitable quasivariational inequality has already been studied in [16-18], in which the authors consider a market of exchange and consumption. In this paper we consider weaker assumptions on the utility function with respect to those considered in the latter mentioned economic models. In particular, we consider upper semicontinuous utility functions, instead of $C^{1}$ ones and, consequently, the differentiability assumption is relaxed. This hypothesis leads to a quasi-variational inequality involving a multivalued map.

More precisely, an economic market with $l$ different goods, $n$ consumers, and $m$ producers will be here considered. Each consumer has a starting endowment of goods and receives a given fraction of the total production, determined by a system of fixed weights. To each commodity 
a nonnegative price not less than a fixed minimum price is associated. The purpose of the market is to determine a price, a production, and a consumption equilibrium: the production equilibrium maximizes the profit of producers and simultaneously the consumption equilibrium maximizes the preferences of consumers under a natural budget constraint. Furthermore, these chooses are made so that the total consumption does not exceed the total production plus the total endowment. Mathematically, the preferences are represented by a utility function, that is, assumed to be convex, proper, and upper semi-continuous, and not necessarily monotone and/or differentiable. We further suppose that the production set is closed, bounded, and convex; namely, producers, cannot have an infinite production.

Finally, we would like to stress that in the classical literature the so-called survivability assumption is required: each agent has, at the beginning, a positive quantity of each commodity (see, e.g., [19]). Thanks to the used variational approach, the novelty of the current paper is mainly to weaken the described survivability assumption. In fact, in this market there is the possibility that, at the beginning, some agent is not endowed with some goods. In this situation, it is required that at least one of the goods owned by the agent must have minimum price not zero; namely, this commodity cannot be a free good. In this way, for any current price the agent has always the opportunity to earn on the sale of its endowment; hence, he can survive in the market.

The plan of the paper is as follows. Firstly, for the reader's convenience, we recall some basic definitions and properties which will be useful in the sequel. After, we introduce the competitive economic equilibrium model integrated with exchange, consumption, and production and we reformulate it in terms of a generalized quasi-variational inequality. Finally, we use this approach to investigate the existence of equilibrium.

It is worth to mention that this model allows to consider a wide class of utility functions, which are only convex, proper, and upper semi-continuous.

\section{Preliminaries}

In the whole section $X, Y$, and $Z$ are Banach spaces. We denote with $B_{X}(x, \eta)$ the ball in $X$ centered at $x$ and of radius $\eta$.

A multivalued map (multimap) $F: X \multimap Y$ is said to be

(a) upper semicontinuous(u.s.c.) if for any $x \in X$ and for any neighborhood $\mathcal{U}$ of $F(x)$ there exists $\eta>0$ such that for all $x^{\prime} \in B_{X}(x, \eta), F\left(x^{\prime}\right) \subset \mathcal{U}$;

(b) lower semicontinuous (l.s.c.) if for any $x \in X$, for any sequence of elements $\left\{x_{n}\right\}_{n=1}^{\infty} \subset X, x_{n} \rightarrow x$, and for any $y \in F(x)$, there exists a sequence of elements $\left\{y_{n}\right\}_{n=1}^{\infty} \subset Y$, with $y_{n} \in F\left(x_{n}\right)$ for all $n, y_{n} \rightarrow y$;

(c) closed if for any sequences $\left\{x_{n}\right\}_{n=1}^{\infty} \subset X,\left\{y_{n}\right\}_{n=1}^{\infty} \subset Y$, if $x_{n} \rightarrow x$ and $y_{n} \in F\left(x_{n}\right), y_{n} \rightarrow y$ then $y \in F(x)$;

(d) compact if its range $F(X)$ is relatively compact in $Y$; that is, $\overline{F(X)}$ is compact in $Y$; (e) quasicompact if its restriction to any compact subset $M \subset X$ is compact.

We recall two results useful to obtain upper semicontinuity and lower semicontinuity (see, e.g., [20] Theorem 1.1.5 and Corollary 1.2.4.).

Theorem 1. Let $F: X \multimap Y$ be a closed and quasicompact multimap. Then $F$ is u.s.c.

Theorem 2. Assume that

(i) $f: X \times Z \rightarrow Y$ is a continuous map such that $f(x, \cdot)$ : $Z \rightarrow Y$ is affine for every $x \in X$;

(ii) $T: X \multimap Y$ and $U: X \multimap Z$ are l.s.c. multimaps with closed and convex values;

(iii) for every $x \in X$ there exists $u \in U(x)$ such that $f(x, u) \in \operatorname{int} T(x)$;

Then the multimap $R: X \multimap Z$ defined by

$$
R(x)=\{u \in U(x): f(x, u) \in T(x)\}
$$

is l.s.c. with closed and convex values.

Definition 3. Let $f: X \rightarrow \mathbb{R}$ be a convex map. For an arbitrary $\bar{x} \in X$ the set $\partial f(\bar{x})$ of all continuous linear functionals $l$ on $X$ with

$$
f(x) \geq f(\bar{x})+l(x-\bar{x}) \quad \forall x \in X
$$

is called the subdifferential of $f$ at $\bar{x}$. A continuous linear functional $l \in \partial f(\bar{x})$ is called a subgradient of $f$ at $\bar{x}$.

Here it follows some properties of the subdifferential.

Proposition 4 (see, e.g., [21] Propositions 2.1.2. and 2.1.5). Let $f: \mathbb{R}^{n} \rightarrow \mathbb{R}$ be a convex and l.s.c. map. Then

(1) for $x \in \mathbb{R}^{n}, \partial f(x)$ is a nonempty convex and compact set;

(2) $\partial f: \mathbb{R}^{n} \multimap \mathbb{R}^{n}$ is an u.s.c. multimap.

To prove our main theorem we will rely on the following existence result for generalized quasi-variational inequalities.

Theorem 5 (see, e.g., [22] Corollary 3.1). Let $f$ and $K$ be multimaps from $\mathbb{R}^{n}$ into itself. Suppose that there exists a nonempty, compact, and convex set $C$ such that

(i) $K(C) \subseteq C$;

(ii) $f$ is a nonempty, contractible, compact valued, and upper semicontinuous multimap on $C$;

(iii) $K$ is a nonempty, continuous, and convex valued multimap on $C$.

Then there exist $x^{\prime} \in K\left(x^{\prime}\right)$ and a vector $y \in f\left(x^{\prime}\right)$ such that

$$
\left\langle y, x-x^{\prime}\right\rangle \leq 0 \quad \forall x \in K\left(x^{\prime}\right)
$$




\section{Equilibrium Model}

We consider a marketplace consisting of two types of agents: $n$ consumers, indexed by $a$, and $m$ producers, indexed by $b$. We denote with $A=\{1, \ldots, n\}, B=\{1, \ldots, m\}$, and $J=\{1, \ldots, l\}$, respectively, sets of consumers, producers, and goods. We denote by $e_{a}^{j}$ and $x_{a}^{j}$ the nonnegative quantities of commodity $j$, respectively, owned and consumed by agent $a$. The vectors $e_{a}=\left(e_{a}^{1}, \ldots, e_{a}^{l}\right) \in \mathbb{R}_{+}^{l}$ and $x_{a}=\left(x_{a}^{1}, \ldots, x_{a}^{l}\right) \in$ $\mathbb{R}_{+}^{l}$ represent, respectively, the initial endowment and the consumption of agent $a$ and $x=\left(x_{1}, \ldots, x_{n}\right) \in \mathbb{R}^{l \times n}$ represents the consumption of the market. For each $a \in A$ we denote by $I_{a}$ the set of indexes corresponding to initial holdings, namely, $I_{a}=\left\{j \in J: e_{a}^{j}>0\right\}$ and we assume that he is endowed with at least one positive commodity, then $I_{a} \neq \emptyset$. We denote by $y_{b}^{j}$ the quantity of commodity $j$ produced by producer $b$. We note that the commodity $y_{b}^{j}$ can also assume negative values: the positive quantity $y_{b}^{j}$ represents the commodity offered in the market by producer $b$, the negative quantity $y_{b}^{j}$ represents the demand required by the market but not satisfied by producer $b$, and $y_{b}^{j}$ equals zero which means that the producer $b$ does not produce the commodity $j$. The vector $y_{b}=\left(y_{b}^{1}, \ldots, y_{b}^{l}\right) \in \mathbb{R}^{l}$ and the matrix $y=\left(y_{1}, \ldots, y_{m}\right) \in \mathbb{R}^{l \times m}$ represent the productions, respectively, of producer $b$ and of the market. To each commodity $j \in J$ is a fixed minimum price $q^{j}$ such that $0 \leq q^{j}<1 / l$. More precisely, each commodity $j$ has a positive price $p^{j}$, which we suppose $p^{j} \geq q^{j}$ for all $j \in J$. We denote by $p=\left(p^{1}, \ldots, p^{l}\right) \in \mathbb{R}_{+}^{l}$ the price vector and we suppose that prices belong to the set

$$
P=\left\{p \in \mathbb{R}_{+}^{l}: \sum_{j=1}^{l} p^{j}=1, p^{j} \geq q^{j} \forall j \in J\right\} .
$$

We observe that as usual in the economic literature, in order to survive in the market, it is required that each agent $a$ is endowed with each commodity $j$, that is, $I_{a}=J$. Thanks to our variational approach and by introduction of the minima prices, we can weaken this assumption by requiring the following weak survivability assumption:

"for all agents $a \in A$ there exists $j \in J$ such that $q^{j} \neq 0$ and $e_{a}^{j} \neq 0$."

Each agent $a$ is endowed with at least one commodity $e_{a}^{j}$ with minimum price greater than zero, namely, $q^{j}>0$. From an economic point of view this means that even if the agent is not endowed with some goods, he can be active in the market. In fact each agent has always the opportunity to earn on the sale of its endowment $\left\langle p, e_{a}\right\rangle>0$.

We denote by $Y_{b}$ the production set of producer $b$, where $Y_{b}$ is assumed to be, as usual, a closed, bounded, convex set of $\mathbb{R}^{l}$ with $0_{\mathbb{R}^{l}} \in Y_{b}$. The boundedness of $Y_{b}$ means that producers cannot have an infinite production and $0_{\mathbb{R}^{l}} \in Y_{b}$ includes the possibility that there is no activity for producer $b$. We indicate with $Y=\prod_{b \in B} Y_{b}$ the total market production. The total production $\sum_{b \in B} y_{b}^{j}$ of commodity $j$ is shared between consumers: each consumer $a$ receives the given fraction $\sum_{b \in B} \theta_{a b} y_{b}^{j}$, determined by a system of fixed weights $\theta_{a b} \geq 0$ such that $\sum_{a \in A} \theta_{a b}=1$ for all $b \in B$. Hence, each consumer $a$, relative to commodity $j$, has at command the quantity $e_{a}^{j}+\sum_{b \in B} \theta_{a b} y_{b}^{j}$. We note that $y_{b}^{j}$ can assume negative values, then it is possible that, the holdings of consumer $a, e_{a}^{j}+\sum_{b \in B} \theta_{a b} y_{b}^{j}$ is negative. Given a price vector $p$, the inner product $\left\langle p, x_{a}\right\rangle$ represents the value of the consumption plan $x_{a},\left\langle p, y_{b}\right\rangle$ represents the value of the production plan $y_{b}$, and $\left\langle p, e_{a}\right\rangle+\max \left\{0,\left\langle p, \sum_{b \in B} \theta_{a b} y_{b}\right\rangle\right\}$ represents the wealth of consumer $a$ at the going prices.

We notice that in the considered model, when the required demand of a commodity is not satisfied, the consumer does not suffer any loss. In this market producers act to maximize their profit $\left\langle p, y_{b}\right\rangle$, while consumers act to maximize their preferences described by a utility function $u_{a}: \mathbb{R}_{+}^{l} \rightarrow \mathbb{R}$, subject to budget constraints: the value of the consumption plan of agent $a$ cannot exceed the agent's wealth at the going prices. The multimap $M_{a}: P \times Y \multimap \mathbb{R}_{+}^{l}$ defined as

$$
\begin{aligned}
& M_{a}(p, y) \\
& \quad=\left\{x_{a} \in \mathbb{R}_{+}^{l}:\left\langle p, x_{a}\right\rangle \leq\left\langle p, e_{a}\right\rangle+\max \left\{0,\left\langle p, \sum_{b \in B} \theta_{a b} y_{b}\right\rangle\right\}\right\}
\end{aligned}
$$

represents the budget constraint set. Previous arguments lead to the following definition of competitive equilibrium.

Definition 6. Letting $\bar{p} \in P, \bar{x} \in \mathbb{R}_{+}^{l \times n}$ and $\bar{y} \in Y$, we say that $(\bar{p}, \bar{x}, \bar{y})$ is a competitive equilibrium if and only if

$$
\begin{aligned}
& \forall b \in B \quad\left\langle\bar{p}, \bar{y}_{b}\right\rangle=\max _{y_{b} \in Y_{b}}\left\langle\bar{p}, y_{b}\right\rangle, \\
& \forall a \in A \quad u_{a}\left(\bar{x}_{a}\right)=\max _{M_{a}(\bar{p}, \bar{y})} u_{a}\left(x_{a}\right), \\
& \forall j \in J \quad \sum_{a \in A}\left(\bar{x}_{a}^{j}-e_{a}^{j}\right)-\sum_{b \in B} \bar{y}_{b}^{j} \leq 0 .
\end{aligned}
$$

We consider the equilibrium problem under the following assumptions:

(H1) for all $b \in B, Y_{b}$ is a closed, bounded, convex set of $\mathbb{R}^{l}$ with $0_{\mathbb{R}^{l}} \in Y_{b}$;

(H2) for all $a \in A, u_{a}$ is concave, proper, and upper semicontinuous.

Denoting with $S: P \times \mathbb{R}_{+}^{l \times n} \times Y \multimap P \times \mathbb{R}_{+}^{l \times n} \times Y$ the multimap defined as $S(p, x, y)=P \times \prod_{a \in A} M_{a}(p, y) \times Y$, we give now a characterization of the competitive equilibrium in terms of the following generalized quasi-variational inequality: 
"find $(\bar{p}, \bar{x}, \bar{y}) \in S(\bar{p}, \bar{x}, \bar{y})$ and $h=\left\{h_{a}\right\}_{a \in A}$, with $h_{a} \in$ $\partial\left(-u_{a}\left(\bar{x}_{a}\right)\right)$ for all $a \in A$ :

$$
\begin{array}{r}
\sum_{b \in B}\left\langle\bar{p}, y_{b}-\bar{y}_{b}\right\rangle+\sum_{a \in A}\left\langle-h_{a}, x_{a}-\bar{x}_{a}\right\rangle \\
+\left\langle\sum_{a \in A}\left(\bar{x}_{a}-e_{a}\right)-\sum_{b \in B} \bar{y}_{b}, p-\bar{p}\right\rangle \leq 0 \\
\forall(p, x, y) \in S(\bar{p}, \bar{x}, \bar{y}) . ”
\end{array}
$$

Theorem 7. Let assumptions (H1)-(H2) be satisfied. A solution to (9), $(\bar{p}, \bar{x}, \bar{y})$, such that

$$
\left\langle\sum_{a \in A}\left(\bar{x}_{a}-e_{a}\right)-\sum_{b \in B} \bar{y}_{b}, \bar{p}-q\right\rangle \leq 0,
$$

is a competitive equilibrium, where $q=\left(q^{1}, \ldots, q^{l}\right)$ is the vector of minima prices.

Proof. We divide the proof in several steps.

Step 1. $(\bar{p}, \bar{y}, \bar{x}) \in S(\bar{p}, \bar{x}, \bar{y})$ and $h_{a} \in\left\{\partial\left(-u_{a}\left(\bar{x}_{a}\right)\right)\right\}$ are solutions to the generalized quasi-variational inequality (9) if and only if, for all $b \in B, \bar{y}_{b}$ is a solution to

$$
\left\langle\bar{p}, y_{b}-\bar{y}_{b}\right\rangle \leq 0, \quad \forall y_{b} \in Y_{b},
$$

for all $a \in A, \bar{x}_{a}$ and $h_{a}$ are solutions to

$$
\left\langle-h_{a}, x_{a}-\bar{x}_{a}\right\rangle \leq 0, \quad \forall x_{a} \in M_{a}(\bar{p}, \bar{y}),
$$

and $\bar{p}$ is a solution to

$$
\left\langle\sum_{a \in A}\left(\bar{x}_{a}-e_{a}\right)-\sum_{b \in B} \bar{y}_{b}, p-\bar{p}\right\rangle \leq 0, \quad \forall p \in P .
$$

This is easily seen by testing (9), respectively, with $(\bar{p}, y, \bar{x})$ for $y \in Y$ such that

$$
y_{s}= \begin{cases}\bar{y}_{s} & \text { if } s \neq b, \\ y_{b} & \forall y_{b} \in Y_{b},\end{cases}
$$

with $(\bar{p}, \bar{y}, x)$ for $x \in \prod_{a \in A} M_{a}(\bar{p}, \bar{y})$ such that

$$
x_{s}= \begin{cases}\bar{x}_{s} & \text { if } s \neq a, \\ x_{a} & \forall x_{a} \in M_{a}(\bar{p}, \bar{y}),\end{cases}
$$

and with $(p, \bar{y}, \bar{x})$ for all $p \in P$. Vice versa, let $\bar{p}, \bar{x}$, and $\bar{y}$ satisfy (11), (12), and (13), then (9) is verified.

Step 2. The maximization problem (6) is equivalent to the variational inequality (11).

It follows directly from the definition of the variational inequality (11).

Step 3. For all $a \in A, \bar{x}_{a} \in M_{a}(\bar{p}, \bar{y})$ is a solution to maximization problem

$$
u_{a}\left(\bar{x}_{a}\right)=\max _{M_{a}(\bar{p}, \bar{y})} u_{a}\left(x_{a}\right), \quad \forall a \in A
$$

if and only if there exists $h_{a} \in \partial\left(-u_{a}\left(\bar{x}_{a}\right)\right)$ such that

$$
\left\langle-h_{a}, x_{a}-\bar{x}_{a}\right\rangle \leq 0 \quad \forall x_{a} \in M_{a}(\bar{p}, \bar{y}) .
$$

It is well known that from $(\mathrm{H} 2)-u_{a}$ is locally Lipschitz continuous. Hence, there exists the generalized directional derivative, $\left(-u_{a}\right)^{0}$, of $-u_{a}$ at $\bar{x}_{a}$ on the direction $\left(x_{a}-\bar{x}_{a}\right)$ and it holds

$$
\begin{aligned}
& \left(-u_{a}\right)^{0}\left(\bar{x}_{a}, x_{a}-\bar{x}_{a}\right) \\
& \quad=\max \left\{\left\langle h_{a}, x_{a}-\bar{x}_{a}\right\rangle: h_{a} \in \partial\left(-u_{a}\left(\bar{x}_{a}\right)\right)\right\} .
\end{aligned}
$$

Furthermore, since $-u_{a}$ is convex, there exists the directional derivative $\left(-u_{a}\right)^{\prime}\left(\bar{x}_{a}, x_{a}-\bar{x}_{a}\right)$; then by (18) we have

$$
\begin{aligned}
& \left(-u_{a}\right)^{0}\left(\bar{x}_{a}, x_{a}-\bar{x}_{a}\right)=\left(-u_{a}\right)^{\prime}\left(\bar{x}_{a}, x_{a}-\bar{x}_{a}\right) \\
& \quad=\max \left\{\left\langle h_{a}, x_{a}-\bar{x}_{a}\right\rangle: h_{a} \in \partial\left(-u_{a}\left(\bar{x}_{a}\right)\right)\right\} .
\end{aligned}
$$

Thus, if $\bar{x}_{a}$ is a minimal point of $-u_{a}$, it follows that

$$
\begin{aligned}
&\left(-u_{a}\right)^{\prime}\left(\bar{x}_{a}, x_{a}-\bar{x}_{a}\right) \\
&=\max \left\{\left\langle h_{a}, x_{a}-\bar{x}_{a}\right\rangle: h_{a} \in\right.\left.\partial\left(-u_{a}\left(\bar{x}_{a}\right)\right)\right\} \geq 0, \\
& \forall x_{a} \in M_{a}(\bar{p}, \bar{y}),
\end{aligned}
$$

then there exists $h_{a} \in \partial\left(-u_{a}\left(\bar{x}_{a}\right)\right)$ such that (17) holds.

Conversely, let $h_{a} \in \partial\left(-u_{a}\left(\bar{x}_{a}\right)\right)$ be such that (17) holds, then

$$
\begin{array}{r}
\left(-u_{a}\right)^{\prime}\left(\bar{x}_{a}, x_{a}-\bar{x}_{a}\right) \\
=\max \left\{\left\langle h_{a}, x_{a}-\bar{x}_{a}\right\rangle: h_{a} \in \partial\left(-u_{a}\left(\bar{x}_{a}\right)\right)\right\} \geq 0, \\
\forall x_{a} \in M_{a}(\bar{p}, \bar{y}) .
\end{array}
$$

From the convexity of $-u_{a}$ it follows that $\varphi(t)=\left(-u_{a}\left(\bar{x}_{a}+\right.\right.$ $\left.\left.t\left(x_{a}-\bar{x}_{a}\right)\right)+u_{a}\left(\bar{x}_{a}\right)\right) / t$ is an increasing function, implying $\left(-u_{a}\right)^{\prime}\left(\bar{x}_{a}, x_{a}-\bar{x}_{a}\right)=\inf _{t>0} \varphi(t)$ and

$$
-u_{a}\left(\bar{x}_{a}+t\left(x_{a}-\bar{x}_{a}\right)\right)+u_{a}\left(\bar{x}_{a}\right) \geq t\left(-u_{a}\right)^{\prime}\left(\bar{x}_{a}, x_{a}-\bar{x}_{a}\right) \geq 0 .
$$

Hence, we get for $t=1,-u_{a}\left(x_{a}\right) \geq-u_{a}\left(\bar{x}_{a}\right)$ for all $x_{a} \in$ $M_{a}(\bar{p}, \bar{y})$. Consequently, $\bar{x}_{a}$ is a maximum point of $u_{a}$ in $M_{a}(\bar{p}, \bar{y})$.

Step 4 . The equilibrium condition (8) holds.

Indeed, by Step 1, variational inequality (13) holds. We pose

$$
\begin{gathered}
J^{+}=\left\{j \in J: \sum_{a \in A}\left(\bar{x}_{a}^{j}-e_{a}^{j}\right)-\sum_{b \in B} \bar{y}_{b}^{j}>0\right\}, \\
J_{0}^{-}=\left\{j \in J: \sum_{a \in A}\left(\bar{x}_{a}^{j}-e_{a}^{j}\right)-\sum_{b \in B} \bar{y}_{b}^{j} \leq 0, \bar{p}^{j}=q^{j}\right\}, \\
J^{-}=\left\{j \in J: \sum_{a \in A}\left(\bar{x}_{a}^{j}-e_{a}^{j}\right)-\sum_{b \in B} \bar{y}_{b}^{j} \leq 0, \bar{p}^{j}>q^{j}\right\} .
\end{gathered}
$$


We suppose by contradiction that $J^{+} \neq \emptyset$. We observe that $J^{-}$ is nonempty; in fact, if $J^{-}=\emptyset$, since $\sum_{j \in J} q^{j}<1$, we have $\bar{p} \neq q$, namely, there exists at least one index $j \in J^{+}$such that $\bar{p}^{j}>q^{j}$, then

$$
\begin{aligned}
& \left\langle\sum_{a \in A}\left(\bar{x}_{a}-e_{a}\right)-\sum_{b \in B} \bar{y}_{b}, \bar{p}-q\right\rangle \\
& =\sum_{j \in J^{+}}\left(\sum_{a \in A}\left(\bar{x}_{a}^{j}-e_{a}^{j}\right)-\sum_{b \in B} \bar{y}_{b}^{j}\right)\left(\bar{p}^{j}-q^{j}\right)>0,
\end{aligned}
$$

but this contradicts $(10)$, then $J^{-} \neq \emptyset$. We consider

$$
\widetilde{p}^{j}= \begin{cases}\bar{p}^{j}+\varepsilon & \forall j \in J^{+}, \\ \bar{p}^{j} & \forall j \in J_{0}^{-}, \\ \bar{p}^{j}-\varepsilon \frac{\left|J^{+}\right|}{\left|J^{-}\right|} & \forall j \in J^{-},\end{cases}
$$

where $0<\varepsilon<\min _{j \in J^{-}}\left\{\left(\bar{p}^{j}-q^{j}\right)\left(\left|J^{-}\right| /\left|J^{+}\right|\right)\right\}$. We have for all $j \in J$ that $\widetilde{p}^{j} \geq q^{j}$ and

$$
\begin{aligned}
\sum_{j \in J} \widetilde{p}^{j} & =\sum_{j \in J^{+}}\left(\bar{p}^{j}+\varepsilon\right)+\sum_{j \in J_{0}^{-}} \bar{p}^{j}+\sum_{j \in J^{-}}\left(\bar{p}^{j}-\varepsilon \frac{\left|J^{+}\right|}{\left|J^{-}\right|}\right) \\
& =1+\varepsilon\left|J^{+}\right|-\left|J^{-}\right| \varepsilon \frac{\left|J^{+}\right|}{\left|J^{-}\right|}=1,
\end{aligned}
$$

then $\widetilde{p} \in P$. If we replace $\widetilde{p}$ in (13), it results in

$$
\begin{aligned}
& \left\langle\sum_{a \in A}\left(\bar{x}_{a}-e_{a}\right)-\sum_{b \in B} \bar{y}_{b}, \tilde{p}-\bar{p}\right\rangle \\
& =\varepsilon \sum_{j \in J^{+}}\left(\sum_{a \in A}\left(\bar{x}_{a}^{j}-e_{a}^{j}\right)-\sum_{b \in B} \bar{y}_{b}^{j}\right) \\
& -\varepsilon \frac{\left|J^{+}\right|}{\left|J^{-}\right|} \sum_{j \in J^{-}}\left(\sum_{a \in A}\left(\bar{x}_{a}^{j}-e_{a}^{j}\right)-\sum_{b \in B} \bar{y}_{b}^{j}\right)>0 .
\end{aligned}
$$

But this is false because $\bar{p}$ is a solution to variational inequality (13). Hence, we have the equilibrium condition (8).

Then we can conclude that $(\bar{p}, \bar{x}, \bar{y})$ is a competitive equilibrium.

Proposition 8. If $(\bar{p}, \bar{x}, \bar{y}) \in S(\bar{p}, \bar{x}, \bar{y})$ is a competitive equilibrium and in (10) the equality holds, then it is a solution to (9).
Proof. By equilibrium condition (8), since $p^{j} \geq q^{j}$ for all $j \in$ $J$, we have that for all $p \in P$, it results in

$$
\begin{aligned}
\left\langle\sum_{a \in A}\left(\bar{x}_{a}-e_{a}\right)-\sum_{b \in B} \bar{y}_{b}, p-\bar{p}\right\rangle & \\
= & \left\langle\sum_{a \in A}\left(\bar{x}_{a}-e_{a}\right)-\sum_{b \in B} \bar{y}_{b}, p-q\right\rangle \\
& +\left\langle\sum_{a \in A}\left(\bar{x}_{a}-e_{a}\right)+\sum_{b \in B} \bar{y}_{b}, q-\bar{p}\right\rangle \\
= & \left\langle\sum_{a \in A}\left(\bar{x}_{a}-e_{a}\right)-\sum_{b \in B} \bar{y}_{b}, p-q\right\rangle \leq 0 .
\end{aligned}
$$

Then $\bar{p}$ is a solution to the variational inequality (13). Moreover, by Steps 2 and 3 of Theorem 7 it follows that, for all $b \in B$ and $a \in A, \bar{y}_{b}$ and $\bar{x}_{a}$ are, respectively, solutions to variational inequalities (11) and (12). Hence, $(\bar{p}, \bar{x}, \bar{y})$ is a solution to the variational problem (9).

\section{Existence Theorems}

This section concerns the study of existence of solutions to the generalized quasi-variational inequality (9). Firstly we achieve an existence result for the following generalized quasi-variational inequality:

"find $(\bar{p}, \bar{x}, \bar{y}) \in P \times \prod_{a \in A} K_{a}(\bar{p}, \bar{y}) \times Y$ and $h=\left\{h_{a}\right\}_{a \in A}$ with $h_{a} \in \partial\left(-u_{a}\left(\bar{x}_{a}\right)\right)$, for all $a \in A$ :

$$
\begin{aligned}
\sum_{b \in B}\left\langle\bar{p}, y_{b}-\bar{y}_{b}\right\rangle+ & \sum_{a \in A}\left\langle-h_{a}, x_{a}-\bar{x}_{a}\right\rangle \\
+ & \left\langle\sum_{a \in A}\left(\bar{x}_{a}-e_{a}\right)-\sum_{b \in B} \bar{y}_{b}, p-\bar{p}\right\rangle \leq 0 \\
& \forall(p, x, y) \in P \times \prod_{a \in A} K_{a}(\bar{p}, \bar{y}) \times Y, ”
\end{aligned}
$$

where $K_{a}(p, y)=M_{a}(p, y) \cap \prod_{j=1}^{l}\left[0, \sum_{a \in A} e_{a}^{j}+M\right]$, with $M>$ 0 such that $-M<\sum_{b \in B} y_{b}^{j}<M$ for all $j \in J$ (the existence of $M$ is ensured by the boundedness of $Y_{b}$ for all $b \in B$ ).

Theorem 9. Let assumptions (H1)-(H2) be satisfied. Then there exists a solution of (29).

Proof. We prove that all the hypotheses of the existence result of Theorem 5 are satisfied obtaining the claimed result.

Step 1 . The multimap $f: \mathbb{R}^{l} \times \mathbb{R}_{+}^{l \times n} \times \mathbb{R}^{l \times m} \multimap \mathbb{R}^{l} \times \mathbb{R}^{l \times n} \times \mathbb{R}^{l \times m}$ defined as

$$
f(p, x, y)=\left(\sum_{a \in A}\left(x_{a}-e_{a}\right)+\sum_{b \in B} y_{b},\left\{-h_{a}\right\}_{a=1}^{n},(p, \ldots, p)\right),
$$

with $h_{a} \in \partial\left(-u_{a}\left(x_{a}\right)\right)$ for any $a \in A$, is u.s.c. with compact and convex values. 
Since, from (H2), the map $-u_{a}$ is a convex and l.s.c. map, from Proposition 4 , it follows that the subdifferential $\partial\left(-u_{a}\right)$ is u.s.c. with compact convex values. Furthermore, the other two components of $f$ are single valued continuous maps, thus we can conclude that the multimap $f$ is u.s.c. with compact convex values.

Step 2. The multimap $K: P \times \mathbb{R}_{+}^{l \times n} \times Y \multimap P \times \mathbb{R}_{+}^{l \times n} \times Y$ defined as $K(p, x, y)=S(p, x, y) \cap C$, with $C=P \times \prod_{j=1}^{l}\left[0, \sum_{a \in A} e_{a}^{j}+\right.$ $M] \times Y$, is l.s.c.

Indeed the map $M_{a}: P \times Y \multimap \mathbb{R}_{+}^{l}$ defined in (5) is l.s.c. To prove it we apply Theorem 2 with $X=P \times Y$, $Z=\mathbb{R}_{+}^{l}, f(x, z)=f(p, y, x)=\langle p, x\rangle-\left\langle p, e_{a}\right\rangle+$ $\max \left\{0,\left\langle p, \sum_{b \in B} \theta_{a b} y_{b}\right\rangle\right\}, T(p, y)=(-\infty, 0], U(p, y)=$ $\prod_{j=1}^{l}\left[0, \sum_{a \in A} e_{a}^{j}+M\right]$.

To apply the mentioned theorem we only need to verify that for any $(p, y) \in P \times Y$ there exists $x \in U(p, y)$ such that

$$
\langle p, x\rangle-\left\langle p, e_{a}\right\rangle-\max \left\{0,\left\langle p, \sum_{b \in B} \theta_{a b} y_{b}\right\rangle\right\}<0 .
$$

This follows easily from the fact that

$$
\left\langle p, e_{a}\right\rangle+\max \left\{0,\left\langle p, \sum_{b \in B} \theta_{a b} y_{b}\right\rangle\right\}>0,
$$

where the last inequality is due to the fact that, since there exists $j=1, \ldots, l$ such that $e_{a}^{j}>0, q^{j}>0$ and $p^{j} \geq q^{j}$ for any $j$, we have for any $p \in P$

$$
\left\langle p, e_{a}\right\rangle=\sum_{j \in I_{a}} p^{j} e_{a}^{j}+\sum_{j \notin I_{a}} p^{j} e_{a}^{j}=\sum_{j \in I_{a}} p^{j} e_{a}^{j}>0 .
$$

Finally, the map $K$ is 1.s.c. as cartesian product of l.s.c. multimaps and trivially it has convex values.

Step 3. $K(C) \subseteq C$.

Follows directly from the definition of $C$.

Step 4 . The map $K$ is u.s.c.

First of all we prove that $M_{a}$ is a closed multimap. Indeed let $\left\{p_{n}\right\} \subset P,\left\{y_{n}\right\} \subset Y$, and $\left\{x_{n}\right\}$, with $x_{n} \in M_{a}\left(p_{n}, y_{n}\right)$ for any $n \in \mathbb{N}$, such that $p_{n} \rightarrow p, y_{n} \rightarrow y$, and $x_{n} \rightarrow x$, one has $x \in M_{a}(p, y)$. In fact, since $x_{n} \in M_{a}\left(p_{n}, y_{n}\right)$ we have

$$
x_{n}^{j} \geq 0\left\langle p_{n}, x_{n}\right\rangle-\left\langle p_{n},-e_{a}\right\rangle-\max \left\{0,\left\langle p_{n}, \sum_{b \in B} \theta_{a b} y_{b n}\right\rangle\right\} \leq 0
$$

passing to the limit and we have

$$
x^{j} \geq 0 \quad\langle p, x\rangle-\left\langle p, e_{a}\right\rangle-\max \left\{0,\left\langle p, \sum_{b \in B} \theta_{a b} y_{b}\right\rangle\right\} \leq 0,
$$

so $x_{a} \in M_{a}(p, y)$.

Hence, $K_{a}$ is a closed multimap as intersection of closed multimaps and $K$ is a closed multimap as cartesian product of closed multimaps.
Moreover, from Step 3, $K$ is a compact multimap, thus, by Theorem 1 it is u.s.c.

Hence, we can apply Theorem 5, obtaining a solution of the quasi-variational inequality (29).

Theorem 10. Let assumptions (H1)-(H2) be satisfied, let $(\bar{p}, \bar{x}, \bar{y})$ and $h=\left\{h_{a}\right\}_{a \in A}$ with $h_{a} \in \partial\left(-u_{a}\left(\bar{x}_{a}\right)\right)$, for all $a \in A$, be a solution to (29), such that (10) is satisfied. Then $(\bar{p}, \bar{x}, \bar{y})$ and $h$ are a solution to (9).

Proof. Fix $a \in A$, we prove that $\bar{x}_{a}$ and $h_{a} \in \partial\left(-u_{a}\left(\bar{x}_{a}\right)\right)$ are a solution to the generalized variational inequality

$$
\left\langle-h_{a}, x_{a}-\bar{x}_{a}\right\rangle \leq 0 \quad \forall x_{a} \in M_{a}(\bar{p}, \bar{y}) .
$$

We suppose that there exists $x^{\prime} \in M_{a}(\bar{p}, \bar{y})$ such that $\left\langle-h_{a}, x^{\prime}-\bar{x}_{a}\right\rangle>0$. Since $\bar{x}_{a} \in K_{a}(\bar{p}, \bar{y}) \subseteq M_{a}(\bar{p}, \bar{y})$, $x^{\prime} \in M_{a}(\bar{p}, \bar{y})$, and $M_{a}(\bar{p}, \bar{y})$ are a convex set, then $\tilde{x}=$ $\lambda x^{\prime}+(1-\lambda) \bar{x}_{a} \in M_{a}(\bar{p}, \bar{y})$, for all $\lambda \in(0,1)$. It results in

$$
\begin{aligned}
\left\langle-h_{a}, \tilde{x}-\bar{x}_{a}\right\rangle & =\left\langle-h_{a}, \lambda x^{\prime}+(1-\lambda) \bar{x}_{a}-\bar{x}_{a}\right\rangle \\
& =\lambda\left\langle-h_{a}, x^{\prime}-\bar{x}_{a}\right\rangle>0 \quad \forall \lambda \in(0,1) .
\end{aligned}
$$

Observe that

$$
\begin{aligned}
& \text { if } \begin{aligned}
\left(x^{\prime}\right)^{j}-\bar{x}_{a}^{j} & =0 \text { then } \tilde{x}^{j}-\sum_{a \in A} e_{a}^{j}-M \\
& =\bar{x}_{a}^{j}-\sum_{a \in A} e_{a}^{j}-M \leq 0 ;
\end{aligned} \\
& \text { if }\left(x^{\prime}\right)^{j}-\bar{x}_{a}^{j}<0 \text { then } \tilde{x}^{j}-\sum_{a \in A} e_{a}^{j}-M<0 .
\end{aligned}
$$

Finally if $\left(x^{\prime}\right)^{j}-\bar{x}_{a}^{j}>0$, by (10) we can choose

$$
0<\lambda<\min \left\{1,-\frac{\bar{x}_{a}^{j}-\sum_{a \in A} e_{a}^{j}-M}{\left(x^{\prime}\right)^{j}-\bar{x}_{a}^{j}},\right.
$$

$$
\text { with } \left.j \in J \text { such that }\left(x^{\prime}\right)^{j}-\bar{x}_{a}^{j}>0\right\} \text {. }
$$

obtaining

$$
\begin{aligned}
\tilde{x}^{j}-\sum_{a \in A} e_{a}^{j}-M \\
=\lambda\left[\left(x^{\prime}\right)^{j}-\bar{x}_{a}^{j}\right]+\bar{x}_{a}^{j}-\sum_{a \in A} e_{a}^{j}-M< \\
\quad-\frac{\bar{x}_{a}^{j}-\sum_{a \in A} e_{a}^{j}-M}{\left(x^{\prime}\right)^{j}-\bar{x}_{a}^{j}}\left[\left(x^{\prime}\right)^{j}-\bar{x}_{a}^{j}\right] \\
\quad+\bar{x}_{a}^{j}-\sum_{a \in A} e_{a}^{j}-M=0 .
\end{aligned}
$$

Hence, $\tilde{x} \in \prod_{j \in J}\left[0, \sum_{a \in A} e_{a}^{j}+M\right]$. Then we have that there exists $\tilde{x} \in M_{a}(\bar{p}, \bar{y}) \cap \prod_{j=1}^{l}\left[0, \sum_{a \in A} e_{a}^{j}+M\right]$ such that $\left\langle-h_{a}, \tilde{x}-\right.$ $\left.\bar{x}_{a}\right\rangle>0$, but this contradicts $\bar{x}_{a}$ solution to

$$
\left\langle-h_{a}, x_{a}-\bar{x}_{a}\right\rangle \leq 0, \quad \forall x_{a} \in K_{a}(\bar{p}, \bar{y}),
$$


and by Step 1 in Theorem 7 this contradicts the fact that $(\bar{p}, \bar{x}, \bar{y})$ is a solution to $(29)$.

Then, we can conclude that $\bar{y}$ is a solution to (11), $\bar{x}_{a}$ is a solution to (36), and $\bar{p}$ is a solution to (13); namely, $(\bar{p}, \bar{x}, \bar{y})$ is a solution to (9).

In conclusion, directly from Theorems 9 and 10, it follows the existence result for a competitive equilibrium.

Theorem 11. Let assumptions (H1)-(H2) be satisfied, let $(\bar{p}, \bar{x}, \bar{y})$ and $h=\left\{h_{a}\right\}_{a \in A}$ with $h_{a} \in \partial\left(-u_{a}\left(\bar{x}_{a}\right)\right)$, for all $a \in A$, be a solution to (29) such that (10) is satisfied. Then $(\bar{p}, \bar{x}, \bar{y})$ and $h$ are a competitive equilibrium.

Remark 12. We observe that if each agent is endowed with each commodity $j$, that is, $I_{a}=J$, then it is possible to assume $q^{j}=0$ for all $j \in J$ and (10) is verified for $(\bar{p}, \bar{x}, \bar{y})$ solution to (29).

In fact, for all $b \in B, 0 \in Y_{b}$ it results in $\left\langle\bar{p}, \bar{y}_{b}\right\rangle \geq 0$, then

$$
\max \left\{0,\left\langle\bar{p}, \sum_{b \in B} \theta_{a b} \bar{y}_{b}\right\rangle\right\}=\left\langle\bar{p}, \sum_{b \in B} \theta_{a b} \bar{y}_{b}\right\rangle .
$$

Moreover, since $\bar{x}_{a} \in M_{a}(\bar{p}, \bar{y})$ is a solution to (12), for all $a \in A$, namely,

$$
\left\langle\bar{p}, \bar{x}_{a}\right\rangle \leq\left\langle\bar{p}, e_{a}-\sum_{b \in B} \theta_{a b} \bar{y}_{b}\right\rangle .
$$

Summing for $a \in A$ it follows that

$$
\begin{aligned}
& \left\langle\bar{p}, \sum_{a \in A} \bar{x}_{a}\right\rangle \leq\left\langle\bar{p}, \sum_{a \in A}\left(e_{a}+\sum_{b \in B} \theta_{a b} \bar{y}_{b}\right)\right\rangle, \\
& \left\langle\bar{p}, \sum_{a \in A} \bar{x}_{a}\right\rangle \leq\left\langle\bar{p}, \sum_{a \in A} e_{a}+\sum_{b \in B} \bar{y}_{b} \sum_{a \in A} \theta_{a b}\right\rangle .
\end{aligned}
$$

In conclusion, being $\sum_{a \in A} \theta_{a b}=1$, (10) holds.

Remark 13. If each agent is endowed with all commodities there exists a competitive equilibrium $(\bar{p}, \bar{x}, \bar{y})$.

\section{Summary and Conclusions}

The main result of this paper has been to obtain the existence of a competitive economic equilibrium for a model integrated with exchange, production, and consumption. In order to obtain a wide applicability in the economic framework, care was taken to keep a level of generality on the assumptions of the market. In particular utility functions which are proper, convex, and upper semi-continuous have been considered, hence without assuming any differentiability. These assumptions allow us to consider a wide range of utility functions frequently used in the economic literature.
A class of utility functions most widely used in economics consists of Cobb-Douglas utility functions:

$$
\begin{aligned}
& u_{a}\left(x_{a}^{1}, \ldots, x_{a}^{l}\right) \\
& \quad=\prod_{j \in J}\left(x_{a}^{j}\right)^{\alpha_{a}^{j}}, \quad \forall x_{a} \in \mathbb{R}_{+}^{l}, \text { with } \alpha_{a}^{j} \geq 0, \quad \sum_{j \in J} \alpha_{a}^{j} \leq 1,
\end{aligned}
$$

where $\alpha_{a}^{j}$ indicates the importance which agent $a$ gives to the commodity $j$. This class of utility functions is very much appreciated in economics, thanks to its analytical tractability (see, e.g., [23] for an historical overview on Cobb-Douglas utility functions).

Another very interesting class of utility functions is represented by Constant Elasticity of Substitution (CES) utility functions:

$$
\begin{aligned}
& u_{a}\left(x_{a}^{1}, \ldots, x_{a}^{l}\right) \\
& =\left[\sum_{j \in J}\left(c_{a}^{j}\right)^{1 / s}\left(x_{a}^{j}\right)^{(s-1) / s}\right]^{s /(s-1)} \\
& \forall x_{a} \in \mathbb{R}_{+}^{l}, \text { with } s>0, s \neq 1,
\end{aligned}
$$

where coefficients $c_{a}^{j} \geq 0$ are distribution parameters. These functions are characterized by constant elasticity of substitution $s$ between any two differentiated goods. The CES utility functions were originally introduced by Kenneth Arrow, as a generalization of Cobb-Douglas utility functions.

The functions, before mentioned, satisfy the assumptions we have taken in this paper; in fact they are proper, convex, and upper semi-continuous. However, these functions are not always differentiable on all their domain. They are defined and continuous on all of $\mathbb{R}_{+}^{l}$, but are only differentiable on the interior of $\mathbb{R}_{+}^{l}$ : in particular the Cobb-Douglas utility function is not differentiable when $\alpha_{a}^{j}<1$ while the CES utility function is never differentiable.

To conclude we want to stress that, in our opinion, by using the variational approach, the generalized quasivariational inequalities are especially suitable to handle equilibrium problems for a market of exchange, consumption, and production, for it allows to take into account a wide class of models.

\section{References}

[1] L. Walras, Elements D'Economique Politique Pure, Corbaz, Lausanne, Switzerland, 1874.

[2] A. Wald, "On some systems of equations of mathematical economics," Econometrica, vol. 19, pp. 368-403, 1951.

[3] K. J. Arrow and G. Debreu, "Existence of an equilibrium for a competitive economy," Econometrica, vol. 22, pp. 265-290, 1954.

[4] D. Gale, "The law of supply and demand," Mathematica Scandinavica, vol. 3, pp. 155-169, 1955.

[5] H. Nikaidō, Convex Structures and Economic Theory, Mathematics in Science and Engineering, Academic Press, New York, NY, USA, 1968. 
[6] A. Barbagallo and M. G. Cojocaru, "Dynamic vaccination games and variational inequalities on time-dependent sets," Journal of Biological Dynamics, vol. 4, no. 6, pp. 539-558, 2010.

[7] A. Barbagallo, P. Daniele, and A. Maugeri, "Variational formulation for a general dynamic financial equilibrium problem: balance law and liability formula," Nonlinear Analysis: Theory, Methods \& Applications, vol. 75, no. 3, pp. 1104-1123, 2012.

[8] M. De Luca and A. Maugeri, "Quasi-variational inequalities and applications to equilibrium problems with elastic demand," in Nonsmooth Optimization and Related Topics, F. M. Clarke, V. F. Demyanov, and F. Giannessi, Eds., vol. 43, pp. 61-77, Plenum, New York, NY, USA, 1989.

[9] F. Facchinei and J. S. Pang, Finite-Dimensional Variational Inequalities and Complementarity Problems, Springer Series in Operations Research and Financial Engineering, 2003.

[10] S. Giuffrè, G. Idone, and S. Pia, "Some classes of projected dynamical systems in Banach spaces and variational inequalities," Journal of Global Optimization, vol. 40, no. 1-3, pp. 119-128, 2008.

[11] G. Idone, A. Maugeri, and C. Vitanza, "Topics on variational analysis and applications to equilibrium problems," Journal of Global Optimization, vol. 28, no. 3-4, pp. 339-346, 2004.

[12] A. Maugeri and C. Vitanza, "Time-dependent equilibrium problems," in Pareto Optimality, Game Theory and Equilibria, A. Chinchuluun, A. Migdalas, P. Pardalos, and L. Pitsoulis, Eds., pp. 505-524, Springer, 2007.

[13] A. Nagurney, Network Economics: A Variational Inequality Approach, Kluwer Academic, 1993.

[14] L. Scrimali, "A variational inequality formulation of the environmental pollution control problem," Optimization Letters, vol. 4, no. 2, pp. 259-274, 2010.

[15] A. Jofré, R. T. Rockafellar, and R. J. B. Wets, "Variational inequalities and economic equilibrium," Mathematics of Operations Research, vol. 32, no. 1, pp. 32-50, 2007.

[16] G. Anello, M. B. Donato, and M. Milasi, "A quasi-variational approach to a competitive economic equilibrium problem without strong monotonicity assumption," Journal of Global Optimization, vol. 48, no. 2, pp. 279-287, 2010.

[17] M. B. Donato, M. Milasi, and C. Vitanza, "An existence result of a quasi-variational inequality associated to an equilibrium problem," Journal of Global Optimization, vol. 40, no. 1-3, pp. 87-97, 2008.

[18] M. B. Donato, M. Milasi, and C. Vitanza, "Quasi-variational approach of a competitive economic equilibrium problem with utility function: existence of equilibrium," Mathematical Models \& Methods in Applied Sciences, vol. 18, no. 3, pp. 351-367, 2008.

[19] A. Jofre, R. T. Rockafellar, and R. J.-B. Wets, "A variational inequality scheme for determining an economic equilibrium of classical or extended type," in Variational Analysis and Applications, vol. 79, pp. 553-577, Springer, 2005.

[20] M. I. Kamenskii, V. V. Obukhovskii, and P. Zecca, Condensing Multivalued Maps and Semilinear Differential Inclusions in Banach Space, W. deGruyter, Berlin, Germany, 2001.

[21] F. H. Clarke, Optimization and Nonsmooth Analysis, vol. 5 of Classics in Applied Mathematics, Society for Industrial and Applied Mathematics, Philadelphia, Pa, USA, 2nd edition, 1990.

[22] D. Chan and J. S. Pang, "The generalized quasivariational inequality problem," Mathematics of Operations Research, vol. 7, no. 2, pp. 211-222, 1982.

[23] P. J. Lloyd, "The origins of the von Thunen-Mill-ParetoWicksell-Cobb-Douglas function," History of Political Economy, vol. 33, pp. 1-19, 2001. 


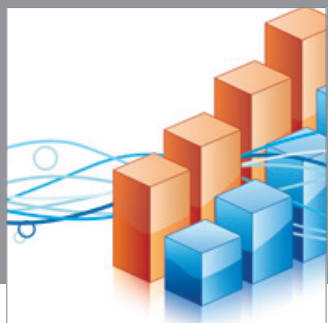

Advances in

Operations Research

mansans

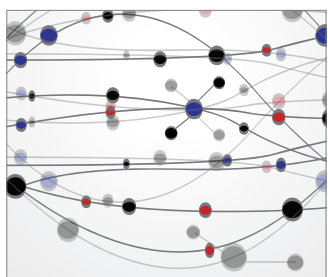

The Scientific World Journal
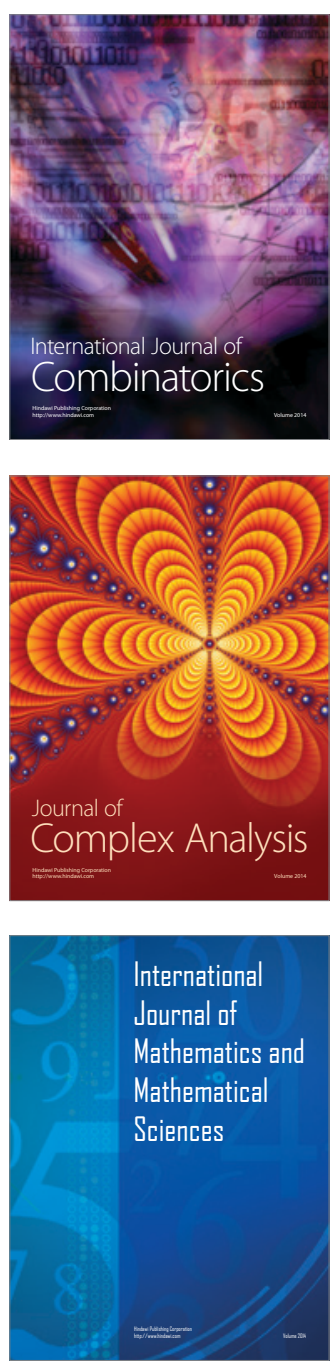
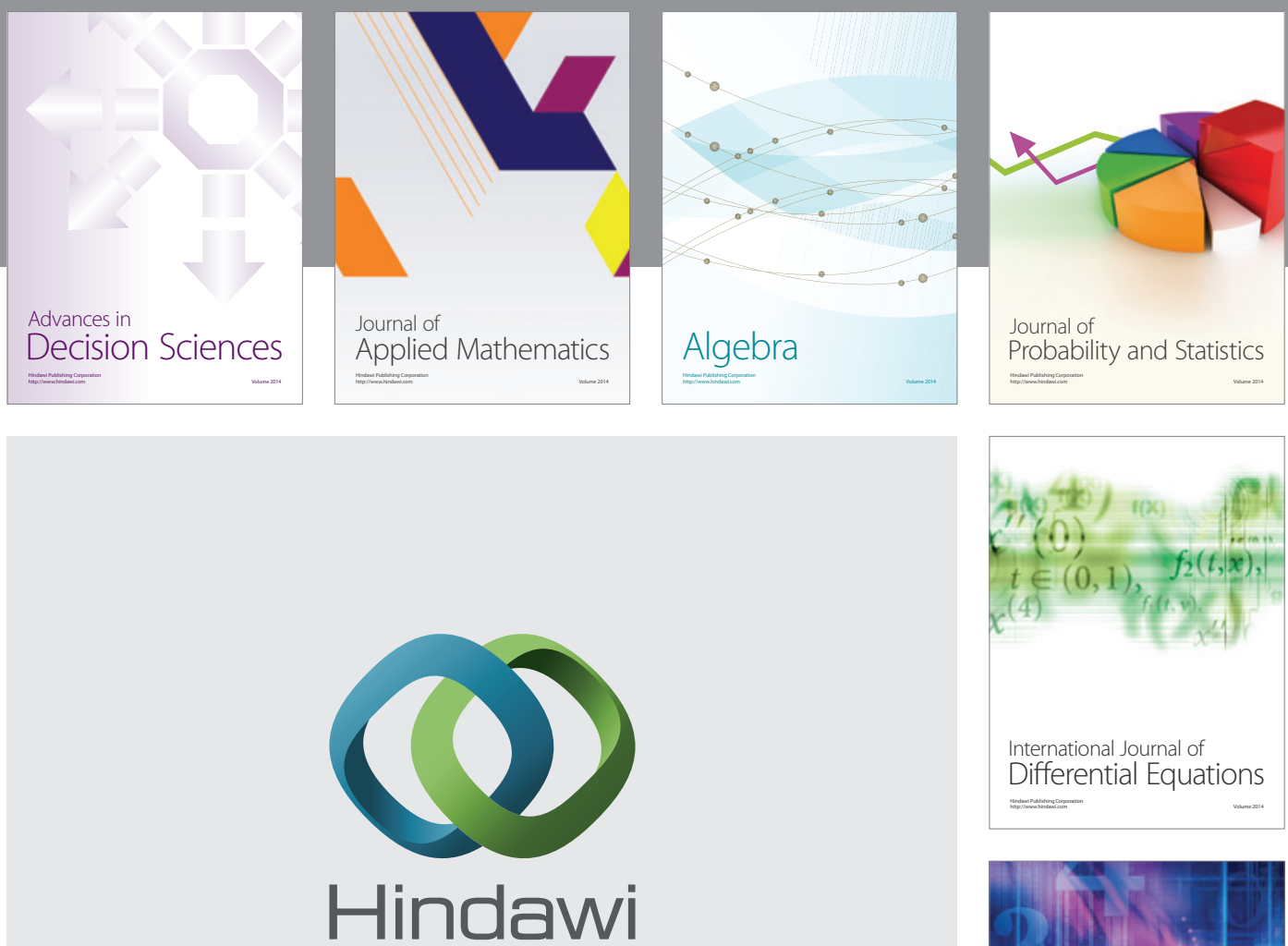

Submit your manuscripts at http://www.hindawi.com
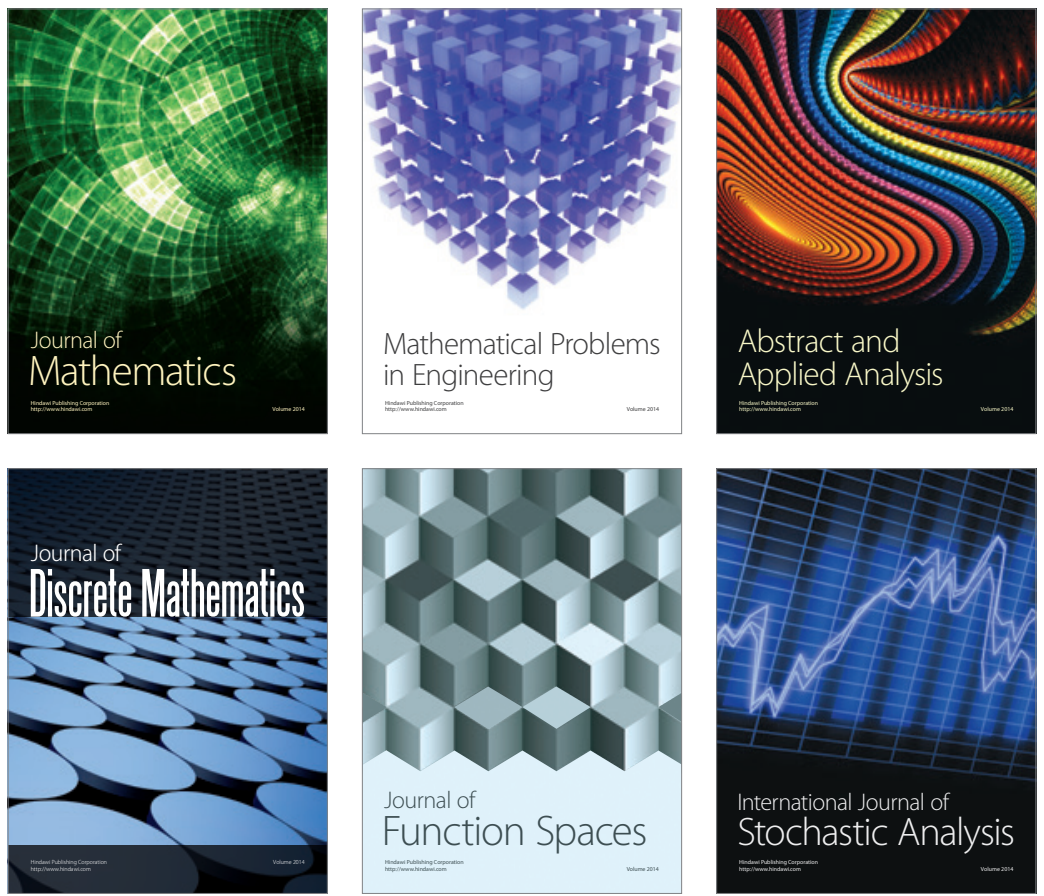

Journal of

Function Spaces

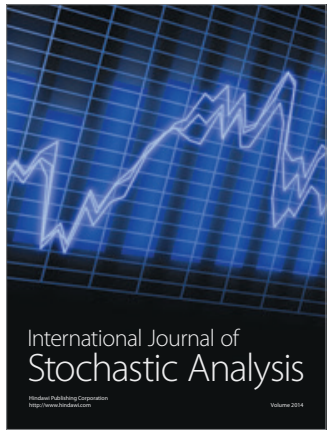

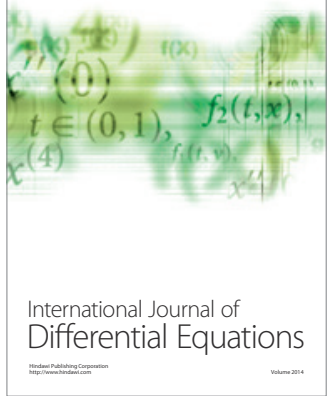
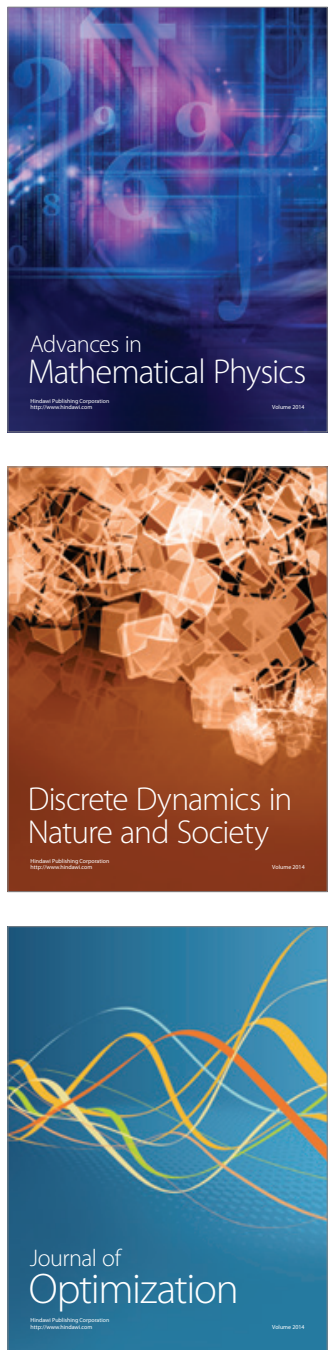